\title{
The complete synthesis of favipiravir from 2-aminopyrazine
}

\author{
Qi Guo ${ }^{1,2} \cdot$ Mingshuo Xu ${ }^{1,2}$. Shuang Guo ${ }^{1,2} \cdot$ Fuqiang Zhu ${ }^{3} \cdot$ Yuanchao Xie ${ }^{1} \cdot$ Jingshan Shen ${ }^{1,2}$
}

Received: 15 June 2018 / Accepted: 23 November 2018 / Published online: 2 January 2019

(c) Institute of Chemistry, Slovak Academy of Sciences 2019

\begin{abstract}
Favipiravir was first synthesized from an inexpensive and commercially available starting material, 2-aminopyrazine. The preferred route embedded within Scheme 4 consisted of seven steps, and was highlighted by the novel and efficient synthesis of 3,6-dichloropyrazine-2-carbonitrile 8. This intermediate was prepared in four successive steps which were regioselective chlorination of the pyrazine ring, bromination, Pd-catalyzed cyanation, and Sandmeyer diazotization/chlorination. This protocol eliminated the hazardous $\mathrm{POCl}_{3}$ of previous synthetic methods and offered a better yield (48\%) which was 1.3 -fold higher than a recently published procedure. From intermediate $\mathbf{8}$, the subsequent nucleophilic fluorination, nitrile hydration and hydroxyl substitution efficiently afforded the target product. Another synthetic approach with the same starting material was also investigated to bypass the allergy-causing dichloro intermediate $\mathbf{8}$. However, the key step of monofluorination at the pyrazine C6 position of intermediate $\mathbf{1 9}$ or $\mathbf{2 2}$ was not achieved.
\end{abstract}

Keywords Favipiravir $\cdot 2$-Aminopyrazine $\cdot$ Fluorination $\cdot$ Sandmeyer reaction

\section{Introduction}

Favipiravir (T-705, 6-fluoro-3-hydroxypyrazine-2-carboxamide) is a novel anti-influenza drug which functions to selectively inhibit the RNA-dependent RNA polymerase of influenza virus (Furuta et al. 2013). T-705 ribofuranosyl triphosphate (T-705 RTP, Fig. 1) is the active form, generated from the parent drug by a series of intracellular enzymes (Sangawa et al. 2013; Naesens et al. 2013). Favipiravir also displays inhibitory activities against a number of other pathogenic RNA viral infections, such as arenavirus,

Electronic supplementary material The online version of this article (https://doi.org/10.1007/s11696-018-0654-9) contains supplementary material, which is available to authorized users.

Yuanchao Xie

xieyuanchao@simm.ac.cn

1 CAS Key Laboratory for Receptor Research, Shanghai Institute of Materia Medica, Chinese Academy of Sciences (CAS), 555 Zuchongzhi Road, Shanghai 201203,

People's Republic of China

2 University of Chinese Academy of Sciences, No. 19A Yuquan Road, Beijing 100049, People's Republic of China

3 Topharman Shanghai Co., Ltd, No.388 Jialilue Road, Zhangjiang Hitech Park, Shanghai 201203, People's Republic of China bunyavirus, flavivirus, alphavirus, and norovirus (Furuta et al. 2013; Furuta et al. 2009; Jin et al. 2015). Moreover, it is believed to be a promising therapeutic candidate for Ebola virus infection (Smither et al. 2014). In a retrospective clinical study during the outbreak of Ebola virus, patients who received T-705 had a significant viral load reduction compared with the control group (Bai et al. 2016). Recently, favipiravir and related structures have attracted extensive attentions in the field of antiviral and antiparasitic research (Gao et al. 2018; Huchting et al. 2018; Huchting et al. 2017; Klejch et al. 2018; Plebanek et al. 2017; Wang et al. 2016).

Favipiravir is a small pyrazine compound containing three substituents $\left(-\mathrm{F},-\mathrm{OH},-\mathrm{CONH}_{2}\right)$ in place. Due to the hydroxyl group, this compound exhibits acidic properties and could be tautomerized to the keto form, shown in Fig. 1 (El-Nahas and Hirao 1999). With respect to the synthesis, three routes are now available. Route 1 is the original one starting from methyl 3-amino-6-bromopyrazine-2-carboxylate 1, and consisted of five steps, diazotization-alcoholysis, Pd-catalyzed imine substitution/hydrolysis, aminolysis, Schiemann fluorination and demethylation (Scheme 1). The first step of diazotization-alcoholysis needs to be performed in concentrated sulfuric acid and only gives a yield of $35 \%$. The following step is a sequence of two reactions, which is tedious and inefficient for the synthesis of $\mathbf{3}$. Moreover, the conversion of $\mathbf{4}$ to $\mathbf{5}$ involves the highly corrosive Olah's 
reagent (Furuta and Egawa 2000). Considering these disadvantages, this route is not welcomed for the scalable production of favipiravir.

Route 2 and Route 3 share the same crucial intermediate, 3,6-dichloropyrazine-2-carbonitrile 8, which is prepared from 3-hydroxypyrazine-2-carboxamide 6 or 3-aminopyrazine-2-carboxylicacid 11 (Schemes 2 and 3). This dichloro-intermediate is then transformed to 9 by reacting with KF, and subsequent nitrile hydration in concentrated hydrochloric acid or treatment with an alkaline solution of $\mathrm{H}_{2} \mathrm{O}_{2}$ gives 10 (Beldar and Jordis 2009; Hara et al. 2010; Liu et al. 2017). The $\mathrm{C} 3$ fluorine of $\mathbf{1 0}$ is more reactive and could be easily replaced by hydroxyl group to furnish the final product. Comparatively, Route 2 is attractive with respect to step count, but not economically feasible for the preparation of $\mathbf{8}$ due to the low yield ( $18 \%$ yield for the nitration of $\mathbf{6}$, shown in the experiment section) and the high cost of the starting material $\mathbf{6}$. Route 3 was reported recently with cheaper 3-aminopyrazine-2-carboxylic acid $\mathbf{1 1}$ as the starting material, from which, five steps were required to obtain $\mathbf{8}$ in a yield of $37 \%$. In both of the routes, a substantial excess of $\mathrm{POCl}_{3}$ and base are indispensable to completely accomplish the functional group transformations. The large<smiles>NC(=O)c1nc(F)cnc1O</smiles>

favipiravir

T-705 RTP, the active form

Fig. 1 Chemical structures of favipiravir (T-705) and T-705 RTP<smiles>COC(=O)c1nc(N)cnc1OC</smiles><smiles>COc1ncc(N)nc1C(N)=O</smiles>

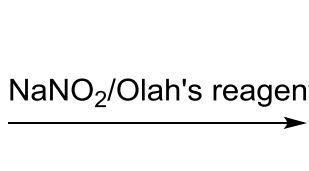<smiles>COc1ncc(F)nc1C(N)=O</smiles>

5<smiles>N=C(N)c1nc(F)cnc1O</smiles>

favipiravir

Scheme 1 Route 1 for the synthesis of favipiravir

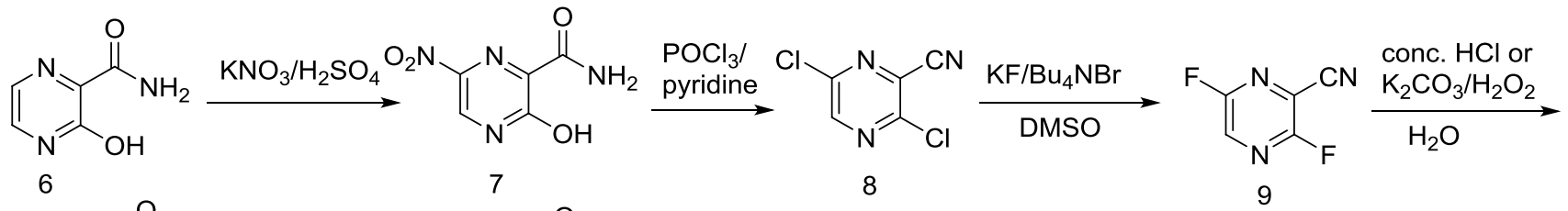<smiles>NC(=O)c1nc(F)cnc1F</smiles>

10<smiles>NC(=O)c1nc(F)cnc1O</smiles>

favipiravir

Scheme 2 Route 2 for the synthesis of favipiravir 


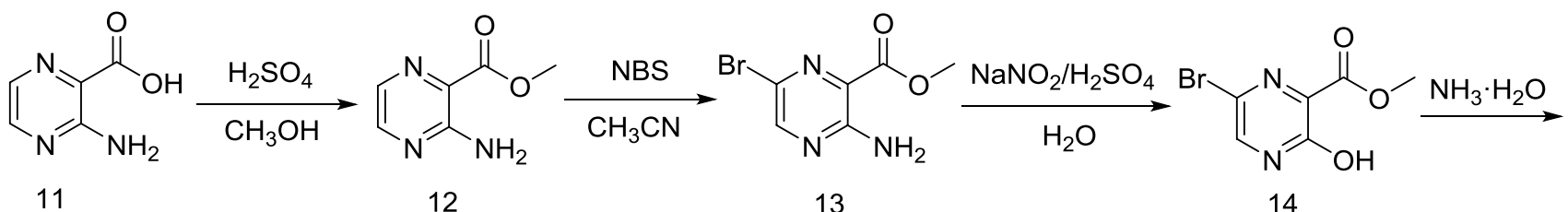<smiles>NC(=O)c1nc(Br)cnc1O</smiles>

15

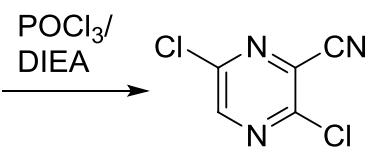

8

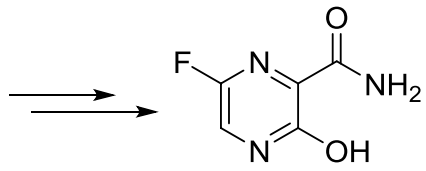

favipiravir

Scheme 3 Route 3 for the synthesis of favipiravir

quantity of $\mathrm{POCl}_{3}$ required in the procedure is a big concern with respect to both scale-up and waste disposal.

Herein, we reported an alternative approach to the synthesis of intermediate 8 without using $\mathrm{POCl}_{3}$. Starting from commercially available 2-aminopyrazine, the synthesis was achieved in $48 \%$ yield over four steps. Following the established procedures, the target compound was smoothly obtained. Another strategy for the synthesis of favipiravir not relying on the allergy-causing dichloro intermediate $\mathbf{8}$ was also investigated. However, no good results were achieved.

\section{Experimental}

\section{Materials and methods}

${ }^{1} \mathrm{H}-\mathrm{NMR}$ and ${ }^{13} \mathrm{C}$-NMR spectra were determined on a Brucker $400 \mathrm{~Hz}$ or Brucker $500 \mathrm{~Hz}$ instrument with TMS as an internal standard. Multiplicities were reported as follows: $\mathrm{s}=$ singlet, $\mathrm{d}=$ doublet, $\mathrm{t}=$ triplet, $\mathrm{q}=$ quartet, $\mathrm{m}=$ multiplet, and $\mathrm{br}=$ broad. ESI-MS was determined on Finnigan ${ }^{\mathrm{TM}}$ LTQ $^{\mathrm{TM}}$ (Thermo Fisher Scientific, Bremen, Germany) linear ion trap mass spectrometer. All the reagents were purchased from commercial suppliers and used without further purification. All reactions were monitored by thin-layer chromatography (TLC) on $25.4 \times 76.2 \mathrm{~mm}$ silica gel plates (GF-254).

\section{Preparation of 2-amino-5-chloropyrazine (17)}

Method A. To a solution of 2-aminopyrazine (1.84 g, $19.4 \mathrm{mmol})$ in acetonitrile $(15 \mathrm{~mL})$ was added NCS $(2.59 \mathrm{~g}, 19.4 \mathrm{mmol})$ in batches at room temperature. The reaction mixture was stirred for $5 \mathrm{~h}$, and filtrated through diatomaceous earth $\left(\right.$ Celite $\left.{ }^{\circledR}\right)$. The filter cake was washed with ethyl acetate $(20 \mathrm{~mL} \times 2)$. The combined filtrate was concentrated under vacuum, and then saturated aqueous
$\mathrm{Na}_{2} \mathrm{CO}_{3}$ solution $(20 \mathrm{~mL})$ was added. The mixture was extracted with ethyl acetate $(100 \mathrm{~mL})$. The organic layer was dried over anhydrous $\mathrm{Na}_{2} \mathrm{SO}_{4}$, decolorized with activated charcoal $(0.5 \mathrm{~g})$, and concentrated. The residue was purified by chromatography on silica gel eluting with petroleum ether (PE)-ethyl acetate (EA) (10:1-2:1) to give a light-yellow solid (1.38 g, yield $55 \%)$.

Method B. To a suspension of 2-aminopyrazine (3 g, $31.6 \mathrm{mmol})$ in acetonitrile $(50 \mathrm{~mL})$ was added $N$-chloro$N$-methoxy-4-methylbenzenesulfonamide (TSA, $8.2 \mathrm{~g}$, $34.7 \mathrm{mmol}$ ) in batches at room temperature. The reaction mixture was heated at $40{ }^{\circ} \mathrm{C}$ for $4 \mathrm{~h}$. Subsequently, the solvent was evaporated in vacuum and the residue was partitioned between ethyl acetate $(50 \mathrm{~mL})$ and saturated aqueous $\mathrm{Na}_{2} \mathrm{CO}_{3}$ solution $(20 \mathrm{~mL})$. The organic layer was dried over anhydrous $\mathrm{Na}_{2} \mathrm{SO}_{4}$, decolorized with activated charcoal ( $0.8 \mathrm{~g})$, and concentrated. The crude product containing $~ 5 \%$ dichlorinated impurity judged by TLC was purified by chromatography on silica gel eluting with $\mathrm{PE} /$ EA (10:1-2:1) to give a light-yellow solid (3.3 g, yield $80 \%)$.

Method C. Reaction of 2-aminopyrazine (3 g, $31.6 \mathrm{mmol}$ ) with $N$-chloro- $N$-methoxybenzenesulfonamide (BSA, $7.7 \mathrm{~g}$, $34.7 \mathrm{mmol}$ ) following the same procedure of method B provided $3.1 \mathrm{~g}$ of $\mathbf{1 7}$ in a yield of $77 \%$.

\section{2-Amino-5-chloropyrazine (17)}

Yield: $80 \%$ (method B), light-yellow solid, M.p.:128$130{ }^{\circ} \mathrm{C}$ (Lit. $129-130{ }^{\circ} \mathrm{C}$, Palamidessi et al. 1964). ${ }^{1} \mathrm{H}$ NMR $\left(400 \mathrm{MHz} \mathrm{CDCl}_{3}\right): \delta 8.01(\mathrm{~d}, 1 \mathrm{H}, J=1.4 \mathrm{~Hz}), 7.76(\mathrm{~d}, 1 \mathrm{H}$, $J=1.4 \mathrm{~Hz}), 4.57(\mathrm{~s}, 2 \mathrm{H}) .{ }^{13} \mathrm{C}$ NMR $\left(125 \mathrm{MHz}, \mathrm{CDCl}_{3}\right): \delta$ 153.19, 141.32, 137.48, 130.82. EI-MS m/z: $129\left(\mathrm{M}^{+}, \mathrm{Cl}^{35}\right.$ 100), $131\left(\mathrm{M}^{+}, \mathrm{Cl}^{37}, 31\right), 102\left(\mathrm{M}^{+}, \mathrm{Cl}^{35},-\mathrm{H},-\mathrm{HCN}, 100\right)$, $104\left(\mathrm{M}^{+}, \mathrm{Cl}^{37},-\mathrm{H},-\mathrm{HCN}, 31\right), 67\left(\mathrm{M}^{+},-\mathrm{H},-\mathrm{HCN},-\mathrm{HCl}\right.$, 28). 


\section{Preparation of 2-amino-3-bromo-5-chloropyrazine} (18a)

$17(1.1 \mathrm{~g}, 8.5 \mathrm{mmol})$ was dissolved in dichloromethane $(25 \mathrm{~mL})$ followed by the addition of NBS $(1.52 \mathrm{~g}, 8.5 \mathrm{mmol})$ in batches over a period of $15 \mathrm{~min}$ at room temperature. When the reaction was completed, saturated aqueous $\mathrm{Na}_{2} \mathrm{CO}_{3}$ solution $(10 \mathrm{~mL})$ was added. After stirring for $10 \mathrm{~min}$, the mixture was partitioned between dichloromethane $(100 \mathrm{~mL})$ and water $(100 \mathrm{~mL})$. The organic layer was dried over anhydrous $\mathrm{Na}_{2} \mathrm{SO}_{4}$, decolorized with activated charcoal $(0.5 \mathrm{~g})$, and concentrated to give a yellow solid $(1.53 \mathrm{~g}$, yield $87 \%)$.

\section{2-Amino-3-bromo-5-chloropyrazine (18a)}

Yield: $87 \%$, yellow solid, M.p.: $108-110{ }^{\circ} \mathrm{C} .{ }^{1} \mathrm{H}$ NMR $\left(400 \mathrm{MHz}, \mathrm{CDCl}_{3}\right): \delta 7.97(\mathrm{~s}, 1 \mathrm{H}), 5.08(\mathrm{~s}, 2 \mathrm{H}) .{ }^{1} \mathrm{H} \mathrm{NMR}$ $\left(400 \mathrm{MHz}, \mathrm{DMSO}-d_{6}\right): \delta 8.10(\mathrm{~s}, 1 \mathrm{H}), 6.99(\mathrm{~s}, 2 \mathrm{H}) .{ }^{13} \mathrm{C}$ NMR (125 MHz, $\left.\mathrm{CDCl}_{3}\right): \delta 151.66,140.26,134.75,123.27$. EI-MS m/z: $207\left(\mathrm{M}^{+}, \mathrm{Cl}^{35}, \mathrm{Br}^{79}, 76\right), 209\left(\mathrm{M}^{+}, \mathrm{Cl}^{37}, \mathrm{Br}^{79}\right.$ and $\left.\mathrm{Cl}^{35}, \mathrm{Br}^{81}, 100\right), 211\left(\mathrm{M}^{+}, \mathrm{Cl}^{37}, \mathrm{Br}^{81}, 26\right), 128\left(\mathrm{M}^{+}\right.$, $\left.\mathrm{Cl}^{35},-\mathrm{Br}, 75\right), 130\left(\mathrm{M}^{+}, \mathrm{Cl}^{37},-\mathrm{Br}, 25\right), 101\left(\mathrm{M}^{+}, \mathrm{Cl}^{35},-\mathrm{Br}\right.$, $-\mathrm{HCN}, 30), 103\left(\mathrm{M}^{+}, \mathrm{Cl}^{37},-\mathrm{Br},-\mathrm{HCN}, 10\right)$.

\section{Preparation of 2-amino-3,5-dibromopyrazine (18b)}

To a suspension of 2-aminopyrazine ( $4 \mathrm{~g}, 42.1 \mathrm{mmol})$ in dichloromethane $(100 \mathrm{~mL})$ was added NBS (18.7 g, $105 \mathrm{mmol}$ ) in batches over a period of $1 \mathrm{~h}$ at room temperature. The reaction mixture was stirred at room temperature for $5 \mathrm{~h}$. When the reaction was completed, saturated aqueous $\mathrm{Na}_{2} \mathrm{CO}_{3}$ solution $(40 \mathrm{~mL})$ was added. After stirring for $15 \mathrm{~min}$, the mixture was partitioned between dichloromethane $(200 \mathrm{~mL})$ and water $(100 \mathrm{~mL})$. The organic layer was then dried over anhydrous $\mathrm{Na}_{2} \mathrm{SO}_{4}$, decolorized with activated charcoal $(1 \mathrm{~g})$, and concentrated to give a yellow solid $(8.0 \mathrm{~g}$, yield $76 \%)$.

\section{2-Amino-3,5-dibromopyrazine (18b)}

Yield: $76 \%$, yellow solid, M.p.: $112-114{ }^{\circ} \mathrm{C}$ (Lit. 113-114 ${ }^{\circ} \mathrm{C}$, Sato et al. 1990a, b). ${ }^{1} \mathrm{H}$ NMR $(400 \mathrm{MHz}$, DMSO- $\left.d_{6}\right): \delta 8.13(\mathrm{~s}, 1 \mathrm{H}), 6.99(\mathrm{~s}, 2 \mathrm{H}) .{ }^{1} \mathrm{H}$ NMR $(400 \mathrm{MHz}$, $\left.\mathrm{CDCl}_{3}\right): \delta 8.04(\mathrm{~s}, 1 \mathrm{H}), 5.09$ (s, 2H). ${ }^{13} \mathrm{C}$ NMR $(125 \mathrm{MHz}$, $\left.\mathrm{CDCl}_{3}\right): \delta 151.86,143.06,123.96,123.65$. ESI-MS $m / z$ : $249.2[\mathrm{M}-\mathrm{H}]^{-}$.

\section{Preparation of 6-chloro-3-aminopyrazine-2- carbonitrile (19a)}

The suspension of 18a ( $3 \mathrm{~g}, 14.5 \mathrm{mmol}), \mathrm{NaCN}(0.85 \mathrm{~g}$, $17.4 \mathrm{mmol}), \mathrm{CuI}(1.3 \mathrm{~g}, 7.3 \mathrm{mmol})$ and $\mathrm{Pd}\left(\mathrm{PPh}_{3}\right)_{4}(0.17 \mathrm{~g}$,
$0.15 \mathrm{mmol}$ ) were stirred in DMF $(40 \mathrm{~mL})$ at $120{ }^{\circ} \mathrm{C}$ for $10 \mathrm{~h}$ under nitrogen protection. After cooling to room temperature, $10 \%$ aqueous solution of $\mathrm{Na}_{2} \mathrm{~S}_{2} \mathrm{O}_{3}(10 \mathrm{~mL})$, water $(60 \mathrm{~mL})$ and ethyl acetate $(200 \mathrm{~mL})$ were added. The resulting mixture was stirred for $10 \mathrm{~min}$, then filtered, and the filter cake was washed with ethyl acetate $(150 \mathrm{~mL})$. The organic layer was separated, washed with water $(3 \times 50 \mathrm{~mL})$, dried over anhydrous $\mathrm{Na}_{2} \mathrm{SO}_{4}$ and concentrated. The residue was purified by chromatography on silica gel eluting with PE/EA (10:1-2:1) to give a light-yellow solid (1.9 g, yield $85 \%)$.

\section{6-Chloro-3-aminopyrazine-2-carbonitrile (19a)}

Yield: $85 \%$, light-yellow solid, M.p.: $156-158{ }^{\circ} \mathrm{C} .{ }^{1} \mathrm{H}$ NMR $\left(400 \mathrm{MHz}, \mathrm{CDCl}_{3}\right): \delta 8.23(\mathrm{~s}, 1 \mathrm{H}), 5.34(\mathrm{~s}, 2 \mathrm{H}) \cdot{ }^{13} \mathrm{C} \mathrm{NMR}$ $\left(100 \mathrm{MHz}, \mathrm{CDCl}_{3}\right): \delta 154.98,146.84,137.41,114.07$, 111.16. EI-MS $m / z: 154\left(\mathrm{M}^{+}, \mathrm{Cl}^{35}, 100\right), 156\left(\mathrm{M}^{+}, \mathrm{Cl}^{37}, 35\right)$, $127\left(\mathrm{M}^{+}, \mathrm{Cl}^{35},-\mathrm{H},-\mathrm{CN}, 48\right), 129\left(\mathrm{M}^{+}, \mathrm{Cl}^{37},-\mathrm{H},-\mathrm{CN}, 15\right)$.

\section{Preparation of 6-bromo-3-aminopyrazine-2- carbonitrile (19b)}

The suspension of $\mathbf{1 8 b}(2.51 \mathrm{~g}, 10.0 \mathrm{mmol}), \mathrm{NaCN}(0.59 \mathrm{~g}$, $12.0 \mathrm{mmol}), \mathrm{CuI}(0.95 \mathrm{~g}, 5.0 \mathrm{mmol})$ and $\mathrm{Pd}\left(\mathrm{PPh}_{3}\right)_{4}(0.14 \mathrm{~g}$, $0.1 \mathrm{mmol})$ were stirred in DMF $(20 \mathrm{~mL})$ at $120{ }^{\circ} \mathrm{C}$ for $10 \mathrm{~h}$ under nitrogen protection. After cooling to room temperature, $10 \%$ aqueous solution of $\mathrm{Na}_{2} \mathrm{~S}_{2} \mathrm{O}_{3}(10 \mathrm{~mL})$ and water $(50 \mathrm{~mL})$ were added. The mixture was extracted with ethyl acetate $(50 \mathrm{~mL} \times 3)$, washed with water $(3 \times 50 \mathrm{~mL})$, dried over anhydrous $\mathrm{Na}_{2} \mathrm{SO}_{4}$ and concentrated. The residue was purified by chromatography on silica gel eluting with PE/ EA (10:1-2:1) to give an off-white solid (1.1 g, yield 55\%).

\section{6-Bromo-3-aminopyrazine-2-carbonitrile (19b)}

Yield: 55\%, off-white solid, M.p.: $180-182{ }^{\circ} \mathrm{C}$ (Lit. 183-184 ${ }^{\circ} \mathrm{C}$, Sato et al. 1990a, b). ${ }^{1} \mathrm{H}$ NMR (400 MHz, DMSO- $\left.d_{6}\right): \delta 8.45$ (s, 1H), 7.62 (s, $\left.2 \mathrm{H}\right) .{ }^{1} \mathrm{H}$ NMR $(400 \mathrm{MHz}$, $\left.\mathrm{CDCl}_{3}\right): \delta 8.30(\mathrm{~s}, 1 \mathrm{H}), 5.28(\mathrm{~s}, 2 \mathrm{H}) \cdot{ }^{13} \mathrm{C}$ NMR $(125 \mathrm{MHz}$, DMSO- $\left.d_{6}\right): \delta 156.70,150.49,123.64,115.45,110.60$. EI-MS $m / z: 198\left(\mathrm{M}^{+}, \mathrm{Br}^{79}, 100\right), 200\left(\mathrm{M}^{+}, \mathrm{Br}^{81}, 98\right), 171$ $\left(\mathrm{M}^{+}, \mathrm{Br}^{79},-\mathrm{H},-\mathrm{CN}, 17\right), 173\left(\mathrm{M}^{+}, \mathrm{Br}^{81},-\mathrm{H},-\mathrm{CN}, 17\right)$, 119( $\left.\mathrm{M}^{+},-\mathrm{Br}, 32\right), 92\left(\mathrm{M}^{+},-\mathrm{Br},-\mathrm{H},-\mathrm{CN}, 46\right)$.

\section{Preparation of 3,6-dichloropyrazine-2-carbonitrile} (8)

To a solution of 19a $(3 \mathrm{~g}, 20 \mathrm{mmol})$ in DCM $(50 \mathrm{~mL})$ at $0{ }^{\circ} \mathrm{C}$ was successively added $\mathrm{TiCl}_{4}(2.2 \mathrm{~mL}, 20 \mathrm{mmol})$ and tertbutyl nitrite $(7.4 \mathrm{~mL}, 62 \mathrm{mmol})$. The reaction mixture was stirred at room temperature for $3 \mathrm{~h}$. When the reaction was completed monitored by TLC, the solvent was evaporated 
and the residue was treated with water $(50 \mathrm{~mL})$ and then extracted with ethyl acetate $(2 \times 50 \mathrm{~mL})$. The extract was dried over $\mathrm{Na}_{2} \mathrm{SO}_{4}$, and concentrated to give $\mathbf{8}$ as a white solid (2.8 g, yield 81\%).

\section{3,6-Dichloropyrazine-2-carbonitrile (8)}

Yield: $81 \%$, white solid, M.p.: $93-94{ }^{\circ} \mathrm{C}$ (Lit. $90-91{ }^{\circ} \mathrm{C}$, Li 2017). ${ }^{1} \mathrm{H}$ NMR (400 MHz, $\left.\mathrm{CDCl}_{3}\right): \delta 8.60(\mathrm{~s}, 1 \mathrm{H}) \cdot{ }^{13} \mathrm{C}$ NMR (125 MHz, $\left.\mathrm{CDCl}_{3}\right): \delta 149.82,147.65,147.03,128.90$, 112.60. EI-MS m/z: $173\left(\mathrm{M}^{+}, \mathrm{Cl}^{35}, \mathrm{Cl}^{35}, 100\right), 175\left(\mathrm{M}^{+}\right.$, $\left.\mathrm{Cl}^{35}, \mathrm{Cl}^{37}, 65\right), 177\left(\mathrm{M}^{+}, \mathrm{Cl}^{37}, \mathrm{Cl}^{37}, 10\right)$.

\section{Preparation of 6-bromo-3-chloropyrazine-2- carbonitrile (20)}

Reaction of 19b (2 g, $10 \mathrm{mmol})$ with $\mathrm{TiCl}_{4}(1.1 \mathrm{~mL}$, $10 \mathrm{mmol})$ and tert-butyl nitrite $(3.7 \mathrm{~mL}, 31 \mathrm{mmol})$ following the above procedure for synthesizing $\mathbf{8}$ gave a white solid $(1.8 \mathrm{~g}$, yield $85 \%)$.

\section{6-Bromo-3-chloropyrazine-2-carbonitrile (20)}

Yield: $85 \%$, white solid, M.p.: $98-100{ }^{\circ} \mathrm{C} .{ }^{1} \mathrm{H}$ NMR (500 MHz, DMSO- $\left.d_{6}\right): \delta 9.08$ (s, 1H). ${ }^{1} \mathrm{H}$ NMR (400 MHz, $\left.\mathrm{CDCl}_{3}\right): \delta 8.71(\mathrm{~s}, 1 \mathrm{H}) .{ }^{13} \mathrm{C}$ NMR $\left(100 \mathrm{MHz}, \mathrm{CDCl}_{3}\right): \delta$ 150.45, 149.82, 138.22, 129.66, 112.58. EI-MS m/z: 217 $\left(\mathrm{M}^{+}, \mathrm{Cl}^{35}, \mathrm{Br}^{79}, 45\right), 219\left(\mathrm{M}^{+}, \mathrm{Cl}^{37}, \mathrm{Br}^{79}\right.$ and $\left.\mathrm{Cl}^{35}, \mathrm{Br}^{81}, 65\right)$, $221\left(\mathrm{M}^{+}, \mathrm{Cl}^{37}, \mathrm{Br}^{81}, 15\right), 138\left(\mathrm{M}^{+}, \mathrm{Cl}^{35},-\mathrm{Br}, 100\right), 140\left(\mathrm{M}^{+}\right.$, $\left.\mathrm{Cl}^{37},-\mathrm{Br}, 30\right), 111\left(\mathrm{M}^{+}, \mathrm{Cl}^{35},-\mathrm{Br},-\mathrm{HCN}, 15\right), 113\left(\mathrm{M}^{+}\right.$, $\left.\mathrm{Cl}^{37},-\mathrm{Br},-\mathrm{HCN}, 4\right)$.

\section{Preparation of 3,6-difluoropyrazine-2-carbonitrile (9)}

A mixture of $8(2.6 \mathrm{~g}, 15.0 \mathrm{mmol}), \mathrm{KF}(5.23 \mathrm{~g}, 90.0 \mathrm{mmol})$, tetrabutylammonium bromide $(1.93 \mathrm{~g}, 6.0 \mathrm{mmol})$ was predried under vacuum over phosphorus pentoxide. The mixture was charged in a polytetrafluoroethylene bottle followed by the addition of dried DMSO $(20 \mathrm{~mL})$, and heated at $60^{\circ} \mathrm{C}$ for $2.5-3 \mathrm{~h}$. The reaction mixture was cooled to room temperature, treated with water $(80 \mathrm{~mL})$ and extracted with diethyl ether $(100 \mathrm{~mL})$. The organic layer was washed with water $(3 \times 25 \mathrm{~mL})$, dried over $\mathrm{Na}_{2} \mathrm{SO}_{4}$, and purified by chromatography on silica gel with PE/EA (50:1-10:1) as eluent to give 9 as a white solid (1.27 g, yield 60\%).

\section{3,6-Difluoropyrazine-2-carbonitrile (9)}

Yield: $60 \%$. white solid, M.p.: $58-60{ }^{\circ} \mathrm{C}$ (Lit. $56-57{ }^{\circ} \mathrm{C}$, Li 2017). ${ }^{1} \mathrm{H}$ NMR (400 MHz, $\left.\mathrm{CDCl}_{3}\right) \delta 8.35$ (dd, $J=8.1$, $1.4 \mathrm{~Hz}, 1 \mathrm{H}) .{ }^{13} \mathrm{C} \mathrm{NMR}\left(125 \mathrm{MHz}, \mathrm{CDCl}_{3}\right): \delta 159.73-157.69$ $(\mathrm{d}, J=255 \mathrm{~Hz}), 157.64-155.62$ (d, $J=252.5 \mathrm{~Hz}), 135.09$ (dd, $J=41.6,11.0 \mathrm{~Hz}), 114.04(\mathrm{~d}, J=35.8 \mathrm{~Hz}), 110.62$ (d, $J=9.1 \mathrm{~Hz})$. EI-MS $m / z: 141\left(\mathrm{M}^{+}, 100\right), 122\left(\mathrm{M}^{+},-\mathrm{F}, 50\right)$, $96\left(\mathrm{M}^{+},-\mathrm{F},-\mathrm{CN}, 50\right)$.

Preparation of 3,6-difluoropyrazine-2-carboxamide (10)

To a solution of $9(1.4 \mathrm{~g}, 10 \mathrm{mmol})$ in THF $(5 \mathrm{~mL})$ was added concentrated hydrochloric acid $(30 \mathrm{~mL})$. The reaction mixture was heated at $60{ }^{\circ} \mathrm{C}$ for $1.5 \mathrm{~h}$, then cooled to room temperature and evaporated under vacuum. The residue was purified by chromatography on silica gel with PE/EA as eluent to give $\mathbf{1 0}$ as a white solid (1.2 $\mathrm{g}$, yield $75 \%$ ).

\section{3,6-Difluoropyrazine-2-carboxamide (10)}

Yield: $75 \%$, white solid, M.p.: $122-124{ }^{\circ} \mathrm{C} .{ }^{1} \mathrm{H}$ NMR $\left(400 \mathrm{MHz}, \mathrm{DMSO}-d_{6}\right) \delta 8.57(\mathrm{dd}, J=8.2,1.7 \mathrm{~Hz}, 1 \mathrm{H})$, $8.21(\mathrm{~s}, 1 \mathrm{H}), 7.98(\mathrm{~s}, 1 \mathrm{H}) .{ }^{13} \mathrm{C}$ NMR $(125 \mathrm{MHz}, \mathrm{DMSO}-$ $\left.d_{6}\right): \delta 162.55(\mathrm{~d}, J=7.2 \mathrm{~Hz}), 157.05-155.08$ (dd, $J=246.4$, $2.5 \mathrm{~Hz}), 156.73-164.69$ (dd, $J=251.7,2.5 \mathrm{~Hz}), 133.46(\mathrm{dd}$, $J=43.2,12.0 \mathrm{~Hz}), 131.50(\mathrm{dd}, J=26.9,8.6 \mathrm{~Hz})$. EI-MS $\mathrm{m} / \mathrm{z}$ : $159\left(\mathrm{M}^{+}, 32\right), 116\left(\mathrm{M}^{+},-\mathrm{CONH}, 100\right), 115\left(\mathrm{M}^{+},-\mathrm{CONH}_{2}\right.$, 25).

\section{Preparation of 6-fluoro-3-hydroxypyrazine-2- carboxamide (favipiravir)}

10 (1.2 g, $7.5 \mathrm{mmol})$, sodium bicarbonate $(3.8 \mathrm{~g}, 45.2 \mathrm{mmol})$ were added into a mixture of 1,4-dioxane $(10 \mathrm{~mL})$ and water $(20 \mathrm{~mL})$. The reaction mixture was heated at $60{ }^{\circ} \mathrm{C}$ for $8 \mathrm{~h}$. When the reaction was completed, hydrochloric acid $(30 \mathrm{~mL}, 3 \mathrm{M})$ was added to adjust the $\mathrm{pH}$ to $3-4$. The precipitate was collected, dried in vacuum to give a lightyellow solid $(0.83 \mathrm{~g})$. The filtrate was extracted with ethyl acetate $(2 \times 50 \mathrm{~mL})$. The organic layer was washed with brine $(2 \times 10 \mathrm{~mL})$, dried over $\mathrm{Na}_{2} \mathrm{SO}_{4}$, and evaporated to give another portion $(0.14 \mathrm{~g})$ of the product. The total yield was $82 \%$.

\section{6-Fluoro-3-hydroxypyrazine-2-carboxamide (favipiravir)}

Yield: $82 \%$, light-yellow solid, M.p.: $176-178{ }^{\circ} \mathrm{C}$ $\left(175-177{ }^{\circ} \mathrm{C}\right.$, Liu et al. 2017). ${ }^{1} \mathrm{H}$ NMR $(400 \mathrm{MHz}$, DMSO- $\left.d_{6}\right): \delta 13.40(\mathrm{~s}, 1 \mathrm{H}), 8.74(\mathrm{~s}, 1 \mathrm{H}), 8.52(\mathrm{~s}, 1 \mathrm{H}), 8.50$ (s, 1H). ${ }^{1} \mathrm{H}$ NMR $\left(400 \mathrm{MHz}, \mathrm{CDCl}_{3}\right): \delta 12.35$ (br, $\left.1 \mathrm{H}\right)$, 8.31 (d, $1 \mathrm{H}, J=8.0 \mathrm{~Hz}), 7.43$ (br, $1 \mathrm{H}), 5.89$ (br, $1 \mathrm{H}) .{ }^{13} \mathrm{C}$ NMR (125 MHz, DMSO- $d_{6}$ ): $\delta 169.20,160.21,152.87$ $(\mathrm{d}, J=243.8 \mathrm{~Hz}), 136.58,122.40$. ESI-MS $\mathrm{m} / \mathrm{z}: 156.1$ $[\mathrm{M}-\mathrm{H}]^{-}$. 


\section{Preparation of 3,5-dibromo-2-hydroxylpyrazine} (21b)

18b (3.0 g, $12 \mathrm{mmol})$ was added into acetic acid $(20 \mathrm{~mL})$ and concentrated sulfuric acid $(4 \mathrm{~mL})$ under ice bath. To this mixture was added a pre-prepared solution of $\mathrm{NaNO}_{2}$ $(1.65 \mathrm{~g}, 24 \mathrm{mmol})$ in water $(10 \mathrm{~mL})$. The reaction mixture was stirred at room temperature for $3 \mathrm{~h}$, and then poured into ice water $(100 \mathrm{~g})$. The precipitate was collected by filtrated, washed with water and dried to give an off-white solid (1.8 g, yield $61 \%)$.

\section{3,5-Dibromo-2-hydroxylpyrazine (21b)}

Yield: $61 \%$, off-white solid, M.p.: $182-184{ }^{\circ} \mathrm{C} .{ }^{1} \mathrm{H}$ NMR (400 $\mathrm{MHz}, \mathrm{CDCl}_{3}$ ): $\delta 11.23(\mathrm{br}, 1 \mathrm{H}), 7.53(\mathrm{~s}, 1 \mathrm{H}) .{ }^{1} \mathrm{H}$ NMR (400 MHz, DMSO- $\left.d_{6}\right): \delta 13.16(\mathrm{~s}, 1 \mathrm{H}), 7.94(\mathrm{~s}, 1 \mathrm{H})$. ${ }^{13} \mathrm{C}$ NMR (125 MHz, $\left.\mathrm{CD}_{3} \mathrm{OD}\right): \delta 154.19,136.73,131.57$, 114.62. ESI-MS m/z: $250.9[\mathrm{M}-\mathrm{H}]^{-}$.

\section{Preparation of 5-chloro-3-bromo-2-hydroxylpyrazine} (21a)

Reaction of $18 \mathbf{a}(3.0 \mathrm{~g}, 14.5 \mathrm{mmol})$ dissolved in acetic acid $(20 \mathrm{~mL})$ and concentrated sulfuric acid $(4 \mathrm{~mL})$ with $\mathrm{NaNO}_{2}(1.9 \mathrm{~g}, 29 \mathrm{mmol})$ in water $(10 \mathrm{~mL})$ following the above procedure for synthesizing 21a gave a yellow solid (1.6 g, yield 54\%).

\section{5-Chloro-3-bromo-2-hydroxylpyrazine (21a)}

Yield: 54\%, yellow solid, M.p.: $184-186{ }^{\circ} \mathrm{C} .{ }^{1} \mathrm{H}$ NMR $\left(400 \mathrm{MHz}, \mathrm{DMSO}-d_{6}\right): \delta 13.11$ (br, $\left.1 \mathrm{H}\right), 7.92(\mathrm{~s}, 1 \mathrm{H})$. ${ }^{1} \mathrm{H}$ NMR $\left(500 \mathrm{MHz}, \mathrm{CD}_{3} \mathrm{OD}\right): \delta 7.58(\mathrm{~s}, 1 \mathrm{H}) .{ }^{13} \mathrm{C} \mathrm{NMR}$ (125 MHz, $\left.\mathrm{CD}_{3} \mathrm{OD}\right): \delta 154.21,136.07,128.96,127.76$. ESI-MS $m / z: 206.9[\mathrm{M}-\mathrm{H}]^{-}$.

\section{Preparation of 6-chloro-3-hydroxylpyrazine-2- carbonitrile (22a)}

Reaction of 21a (1.0 g, $4.8 \mathrm{mmol})$ with $\mathrm{NaCN}$ (280 mg, $5.8 \mathrm{mmol})$ catalyzed by $\mathrm{CuI}(460 \mathrm{mg}, 2.4 \mathrm{mmol})$ and $\mathrm{Pd}\left(\mathrm{PPh}_{3}\right)_{4}(55 \mathrm{mg}, 0.05 \mathrm{mmol})$ according to the procedure for synthesizing 19b gave a gray solid $(0.5 \mathrm{~g}$, yield $64 \%)$.

\section{6-Chloro-3-hydroxylpyrazine-2-carbonitrile (22a)}

Yield: $64 \%$, gray solid, M.p.: $158-160{ }^{\circ} \mathrm{C} .{ }^{1} \mathrm{H}$ NMR $\left(400 \mathrm{MHz}, \mathrm{DMSO}-d_{6}\right): \delta 7.97(\mathrm{~s}, 1 \mathrm{H}) .{ }^{1} \mathrm{H}$ NMR $(500 \mathrm{MHz}$, $\left.\mathrm{CD}_{3} \mathrm{OD}\right): \delta 8.25$ (s, $\left.1 \mathrm{H}\right) .{ }^{13} \mathrm{C} \mathrm{NMR}\left(125 \mathrm{MHz}, \mathrm{CD}_{3} \mathrm{OD}\right)$ : $\delta 160.73,143.18,134.78,118.47,113.87$. ESI-MS $m / z$ : $153.9[\mathrm{M}-\mathrm{H}]^{-}$.

\section{Preparation of 6-bromo-3-hydroxylpyrazine-2- carbonitrile (22b)}

Reaction of $21 \mathbf{b}$ ( $1 \mathrm{~g}, 4 \mathrm{mmol}$ ) with $\mathrm{NaCN}$ (230 mg, $4.8 \mathrm{mmol})$ catalyzed by $\mathrm{CuI}(380 \mathrm{mg}, 2 \mathrm{mmol})$ and $\mathrm{Pd}\left(\mathrm{PPh}_{3}\right)_{4}(50 \mathrm{mg}, 0.04 \mathrm{mmol})$ according to the procedure for synthesizing 19b gave a gray solid (350 mg, yield $45 \%$ ).

\section{6-Bromo-3-hydroxylpyrazine-2-carbonitrile (22b)}

Yield: $45 \%$, gray solid, M.p.: $168-170{ }^{\circ} \mathrm{C} .{ }^{1} \mathrm{H}$ NMR $\left(400 \mathrm{MHz}, \mathrm{DMSO}-d_{6}\right): \delta 8.46(\mathrm{~s}, 1 \mathrm{H}) .{ }^{13} \mathrm{C} \mathrm{NMR}(125 \mathrm{MHz}$, DMSO- $\left.d_{6}\right): \delta 151.12,133.03,129.18,114.82,99.21$. ESI-MS $m / z: 197.9[\mathrm{M}-\mathrm{H}]^{-}$.

\section{Preparation of 3-hydroxy-6-nitropyrazine-2- carboxamide (7)}

To a solution of $6(4.0 \mathrm{~g}, 28.7 \mathrm{mmol})$ in concentrated sulfuric acid $(20 \mathrm{~mL})$ under ice-cooling was added potassium nitrate $(5.8 \mathrm{~g}, 57.5 \mathrm{mmol})$ in three batches. After stirring at $40{ }^{\circ} \mathrm{C}$ for $4 \mathrm{~h}$, the reaction mixture was poured into ice water (200 g). The resulting precipitate was collected by filtration. The solid was slurried in pure water $(20 \mathrm{~mL})$ and dried to give a light-yellow solid (1 g, yield 19\%).

\section{3-Hydroxy-6-nitropyrazine-2-carboxamide (7)}

Yield: $19 \%$, light yellow solid, M.p.: $182-184{ }^{\circ} \mathrm{C} .{ }^{1} \mathrm{H}$ NMR (400 MHz, DMSO- $\left.d_{6}\right): \delta 8.96(\mathrm{~s}, 1 \mathrm{H}), 8.32(\mathrm{~s}, 1 \mathrm{H}), 8.05(\mathrm{~s}$, $1 \mathrm{H}) .{ }^{13} \mathrm{C}$ NMR $\left(125 \mathrm{MHz}, \mathrm{DMSO}-d_{6}\right): \delta 163.60,156.92$, 143.19, 138.63, 134.26. EI-MS m/z: $184\left(\mathrm{M}^{+}, 84\right), 141\left(\mathrm{M}^{+}\right.$, $-\mathrm{CONH}, 10)$.

\section{Results and discussion}

The new synthetic approach to favipiravir starting from 2-aminopyarzine 16 was outlined in Scheme 4. The preferred route (highlighted in red) consisted of seven steps, featured with a novel and efficient method to the synthesis of $\mathbf{8}$ in four transformations.

With respect to the first step, at the beginning, mono-chlorination of 16 using $\mathrm{NCS}$ in $\mathrm{CHCl}_{3}$ was plagued by a very low yield and large amounts of by-products (Cai et al. 2007). After a preliminary solvent screening, we determined acetonitrile as the optimal reaction medium. However, further condition optimizations did not afford significant improvement in product yield, shown in Table 1. A stoichiometric 


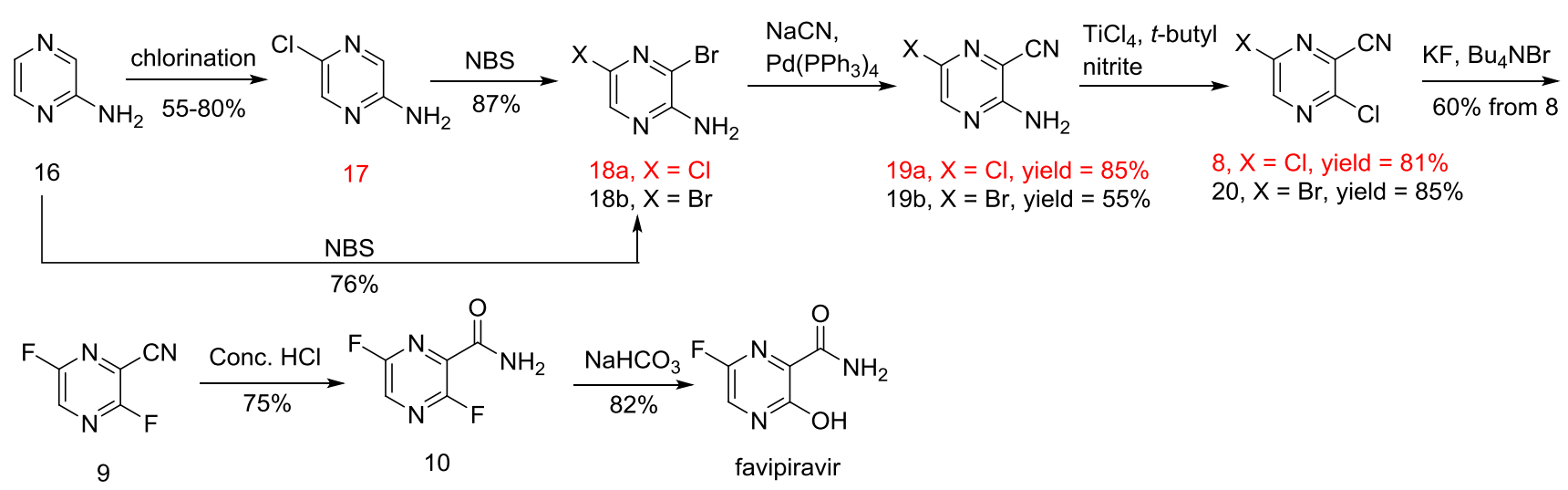

Scheme 4 Our synthetic approach to favipiravir in this paper and the preferred route was highlighted in red

Table 1 Screening of reaction conditions for the mono-chlorination of $\mathbf{1 6}$<smiles>CON(Cl)S(=O)(=O)c1ccc(CCc2ccccc2)cc1S(=O)(=O)N(C)Cl</smiles>

\begin{tabular}{lllccc}
\hline Entry & Chlorinating Reagent & Equivalent $(\mathrm{eq})$ & Time $(\mathrm{h})$ & Temperature $\left({ }^{\circ} \mathrm{C}\right)$ & Yield $(\%)$ \\
\hline 1 & NCS & 0.9 & 8 & R.T. & 44 \\
2 & NCS & 1.0 & 8 & R.T. & R.T. \\
3 & NCS & 1.1 & 8 & R.T. & 50 \\
4 & 1.2 & 8 & 50 & 78 \\
5 & NCS & 1.1 & 12 & 50 & 51 \\
6 & BSA & 0.9 & 12 & 50 & 76 \\
7 & TSA & 1.0 & 12 & 50 & 80 \\
8 & TSA & 1.1 & 12 & 50 & 54 \\
9
\end{tabular}

amount of NCS was not sufficient to react with all the material (Entry 2), and an increase in the amount of NCS would lead to an overchlorinated product (Entry 3-4). Moreover, the reaction mixture became dark brown and cloudy with time, probably due to the formation of polymers (Cai et al. 2007). Recently, Pu et al. reported a chlorinating reagent (BSA) which provided an 80\% yield of 17 (Pu et al. 2016). Accordingly, we prepared BSA as well as the analog TSA, and performed the reaction using the two reagents. 1.1 Equiv of BSA gave the product in a yield of $77 \%$ consistent with the previous study (Table 1, Entry 5). However, BSA was oil, not convenient to use. TSA was a solid and used for screening of reaction conditions. It was found that an increase of TSA from 0.9 to 1.1 equiv significantly improved the yield (Entry 6-8), offering a maximum of $80 \%$. Further increase in the amount of TSA was detrimental to the yield (Entry 9), which was exclusively attributed to the overchlorinated by-product. The optimal ratio of TSA was 1.1 equiv, also producing a small amount $(\sim 5 \%)$ of the overchlorinated impurity, but could be removed at the stage or downstream by chromatographic purification or recrystallization.

17 was amenable to bromination at the $\mathrm{C} 3$ position with NBS in a yield of $87 \%$, and subsequent replacement of the bromine atom by the nitrile group smoothly afforded 19a. Sodium cyanide proved to be a good nitrile donor in the coupling reaction with catalytic $\mathrm{Pd}\left(\mathrm{PPh}_{3}\right)_{4}$. The reaction 
Table 2 Screening of reaction conditions for the preparation of 19a

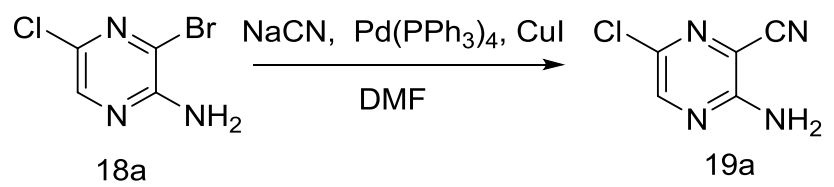

\begin{tabular}{llllll}
\hline Entry & $\mathrm{NaCN}(\mathrm{eq})$ & $\mathrm{Pd}\left(\mathrm{PPh}_{3}\right)_{4}(\mathrm{eq})$ & Time $(\mathrm{h})$ & $\begin{array}{l}\text { Tem- } \\
\text { perature } \\
\left({ }^{\circ} \mathrm{C}\right)\end{array}$ & Yield $(\%)$ \\
\hline 1 & 1.2 & 0.01 & 14 & 80 & 47 \\
2 & 1.2 & 0.01 & 14 & 100 & 52 \\
3 & 1.1 & 0.01 & 10 & 120 & 79 \\
4 & 1.2 & 0.01 & 10 & 120 & 85 \\
5 & 1.2 & 0.01 & 10 & 150 & 72 \\
6 & 1.5 & 0.01 & 10 & 120 & 82 \\
7 & 1.2 & 0.005 & 10 & 120 & 74 \\
8 & 1.2 & 0.02 & 10 & 120 & 82 \\
\hline
\end{tabular}

yield was influenced by several factors, so we made a preliminary investigation of the reaction condition (Table 2). This coupling reaction should be performed at a temperature of about $120{ }^{\circ} \mathrm{C}$. Reducing the temperature to $100{ }^{\circ} \mathrm{C}$ or $80{ }^{\circ} \mathrm{C}$ remarkably decreased the yield even with a prolonged reaction time (Entry 1-2). $1 \mathrm{~mol} \%$ of $\mathrm{Pd}\left(\mathrm{PPh}_{3}\right)_{4}$ were sufficient for the coupling reaction (Entry 4 and Entry 7-8), and the optimal amount of $\mathrm{NaCN}$ was 1.2 equiv (Entry 3-4 and Entry 6). In theory, the chlorine atom at the C6 position of 19a could also be replaced by the nitrile group in the coupling reaction, so it would be better to control the amount of $\mathrm{NaCN}$ in the range of 1.2-1.5 equiv. Generally, the best condition was Entry 5 with 1.2 equiv of $\mathrm{NaCN}, 1 \mathrm{~mol} \%$ of $\operatorname{Pd}\left(\mathrm{PPh}_{3}\right)_{4}$ and $120{ }^{\circ} \mathrm{C}$ of reaction temperature, which provided a yield of $85 \%$ (Entry 4 ).

With compound 19a in hand, it was easy to prepare the key intermediate $\mathbf{8}$ via Sandmeyer reaction. $t$-Butyl nitrite and titanium tetrachloride $\left(\mathrm{TiCl}_{4}\right)$ was applied to make this transformation on account of the simple workup and the good yield $(>80 \%)$. The residual $\mathrm{TiCl}_{4}$ would be converted to the non-toxic titanium dioxide by reacting with water in the post-processing.

Nucleophilic fluorination of 8 with KF (6 equiv) and $\mathrm{Bu}_{4} \mathrm{NBr}$ (2 equiv) gave the difluoro product 9 in $60 \%$ isolated yield. Compound 19 bearing a $\mathrm{C} 5$ bromine group was prepared with the same strategy, but it proved to be a very poor substrate, only giving a small amount of the desired product. For the fluorination step, glass reaction vessel should be avoided, otherwise the yield would be severely decreased. The subsequent acid-mediated nitrile hydration proceeded efficiently and the derived intermediate $\mathbf{1 0}$ was subjected to anhydrous $\mathrm{NaHCO}_{3}$ solution to afford the final product in a yield of $62 \%$ over two steps. The difluoro intermediate $\mathbf{9}$ had a low melting point, and the general workup procedure may easily cause yield loss. Recently, Liu et al. reported a one-pot three-step protocol for the synthesis of favipiravir from intermediate $\mathbf{8}$ in good yield $(60 \%)$. Since intermediates $\mathbf{9}$ and $\mathbf{1 0}$ did not need to be isolated, this process could minimize the yield loss (Liu et al. 2017).

Compound $\mathbf{8}$ is a potential allergy-causing substance, which we had confirmed during the course of our studies. To avoid using this intermediate, another synthetic route was investigated. Retrosynthetically, compound $\mathbf{2 3}$ or $\mathbf{2 4}$, if available, could be a favorable precursor of favipiravir. Along this line, we made our effort to synthesize the two compounds (Scheme 5). Compound 22a and 22b were synthesized from 18 in two steps via Sandmeyer hydroxylation reaction and Pd-catalyzed cyanation of 21. However, reaction of the two compounds with $\mathrm{KF}$ or $\mathrm{CsF}$ under the same condition did not give the desired product and most of the starting material was remained in the reaction mixture. Based on the reaction results from 19, 8, 20 and 22 , it could be concluded that the fluoride preferred to replace the chlorine atom instead of the bromine atom; the adjacent $\mathrm{Cl}$ of the nitrile group of $\mathbf{8}$ was first substituted, which would facilitate the fluorination at the

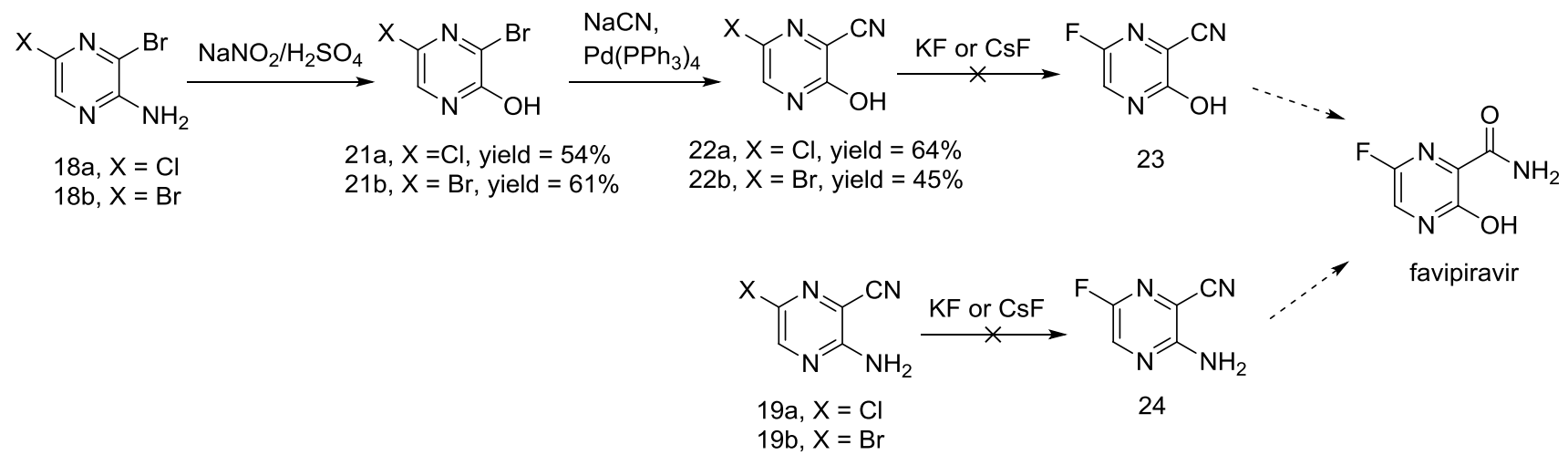

Scheme 5 Another synthetic approach to favipiravir proposed in this paper 
C6 position; and mono-fluorination at the $\mathrm{C} 6$ position of $\mathbf{1 9}$ or $\mathbf{2 2}$ was very difficult.

\section{Conclusion}

In summary, we investigated the synthesis of favipiravir starting from an inexpensive and commercially available 2-aminopyrazine. A facile route for the synthesis of $\mathbf{8}$ was developed in four steps. This new approach was free from the hazardous $\mathrm{POCl}_{3}$ and provided a good yield. The purpose of finding a novel synthesis to favipiravir not via intermediate 8 was not achieved, but due to the allergy-causing problems of $\mathbf{8}$, alternative protocols should be considered.

Acknowledgements This work was supported by National Science Foundation for Young Scientists of China (Grant no. 21502209). The authors gratefully acknowledged Topharman Shanghai Co., Ltd for collaboration.

\section{Compliance with ethical standards}

Conflict of interest On behalf of all authors, the corresponding author states that there is no conflict of interest.

\section{References}

Bai CQ, Mu JS, Kargbo D, Song YB, Niu WK, Nie WM, Kanu A, Liu WW, Wang YP, Dafae F, Yan T, Hu Y, Deng YQ, Lu HJ, Yang F, Zhang XG, Sun Y, Cao YX, Su HX, Sun Y, Liu WS, Wang CY, Qian J, Liu L, Wang H, Tong YG, Liu ZY, Chen YS, Wang HQ, Kargbo B, Gao GF, Jiang JF (2016) Clinical and virological characteristics of Ebola virus disease patients treated with favipiravir (T-705)-Sierra leone, 2014. Clin Infect Dis 6310:1288-1294. https ://doi.org/10.1093/cid/ciw571

Beldar SV, Jordis U (2009) Synthetic studies towards the antiviral pyrazine derivative T-705. Institute of Applied Synthetic Chemistry, Vienna University of Technology, Vienna, p 13

Cai L, Pike V W, Innis R B (2007) (Aminophenyl)imidazo[1,2-a]pyridine derivatives useful as beta-amyloid PET imaging agents and their preparation. WO2007124345A2 (issued November 1, 2007)

El-Nahas A, Hirao K (1999) A theoretical study on 2-hydroxypyrazine and 2,3-dihydroxypyrazine: tautomerism, intramolecular hydrogen bond, solvent effects. J Mol Struct 4591:229-237. https://doi. org/10.1016/s0166-1280(98)00270-x

Furuta Y, Egawa H (2000) Nitrogenous heterocyclic carboxamide derivatives or salts thereof and antiviral agents containing both. European Patent Office WO, 00/10569 (issued March 2, 2000)

Furuta Y, Takahashi K, Shiraki K, Sakamoto K, Smee DF, Barnard DL, Gowen BB, Julander JG, Morrey JD (2009) T-705 (favipiravir) and related compounds: novel broad-spectrum inhibitors of RNA viral infections. Antivir Res 823:95-102. https://doi.org/10.1016/j.antiv iral.2009.02.198

Furuta Y, Gowen BB, Takahashi K, Shiraki K, Smee DF, Barnard DL (2013) Favipiravir (T-705), a novel viral RNA polymerase inhibitor. Antivir Res 1002:446-454. https://doi.org/10.1016/j.antiv iral.2013.09.015

Gao J, Luo X, Li Y, Gao R, Chen H, Ji D (2018) Synthesis and biological evaluation of 2-oxo-pyrazine-3-carboxamide-yl nucleoside analogues and their epimers as inhibitors of influenza a viruses. Chem Bio Drug Des 853:245-252. https://doi.org/10.1111/cbdd.12382
Hara T, Norimatsu N, Kurushima H, Kano T (2010) Method for producing dichloropyrazine derivative. European Patent Office WO2010087117 (issued August 2, 2012)

Huchting J, Winkler M, Nasser H, Meier C (2017) Synthesis of T-705-ribonucleoside and T-705-ribonucleotide and Studies of chemical stability. ChemMedChem 129:652-659. https://doi.org/10.1002/ cmdc. 201700116

Huchting J, Vanderlinden E, Winkler M, Nasser H, Naesens L, Meier C (2018) Prodrugs of the phosphoribosylated forms of hydroxypyrazinecarboxamide Pseudobase T-705 and its de-fluoro analogue T-1105 as potent influenza virus inhibitors. J Med Chem 61:61936210. https://doi.org/10.1021/acs.jmedchem.8b00617

Jin Z, Tucker K, Lin X, Kao CC, Shaw K, Tan H, Symons J, Behera I, Rajwanshi VK, Dyatkina N, Wang G, Beigelman L, Deval J (2015) Biochemical evaluation of the inhibition properties of favipiravir and 2'-C-methyl-cytidine triphosphates against human and mouse norovirus RNA polymerases. Antimicrob Agents Chemother 5912:7504 7516. https://doi.org/10.1128/aac.01391-15

Klejch T, Pohl R, Janeba Z, Sun M, Keough D, Guddat L, Hockova D (2018) Acyclic nucleoside phosphonates with unnatural nucleobases, favipiravir and allopurinol, designed as potential inhibitors of the human and Plasmodium falciparum 6-oxopurine phosphoribosyltransferases. Tetrahedron 74:5886-5897. https://doi. org/10.1016/j.tet.2018.08.014

Li M (2017) Synthetic method of favipiravir. State Intellectual Property Office of the P.R.C. CN107226794A (issued October 3, 2017)

Liu F-L, Li C-Q, Xiang H-Y, Feng S (2017) A practical and step-economic route to Favipiravir. Chem Pap 7111:2153-2158. https://doi. org/10.1007/s11696-017-0208-6

Naesens L, Guddat LW, Keough DT, van Kuilenburg AB, Meijer J, Vande Voorde J, Balzarini J (2013) Role of human hypoxanthine guanine phosphoribosyltransferase in activation of the antiviral agent T-705 (favipiravir). Mol Pharmacol 844:615-629. https://doi.org/10.1124/ mol.113.087247

Palamidessi G, Bernardi L (1964) On 2, 5-dichloropyrazine. J Org Chem 298:2491-2492

Plebanek E, Lescrinier E, Andrei G, Snoeck R, Herdewijn P, Jonghe SD (2017) Emimycin and its nucleoside derivatives: synthesis and antiviral activity. Eur J Med Chem 144:93-103. https://doi. org/10.1016/j.ejmech.2017.12.018

$\mathrm{Pu}$ X, Li Q, Lu Z, Yang X (2016) $N$-Chloro- $N$-methoxybenzenesulfonamide: a chlorinating reagent. Eur J Org Chem 36:5937-5940. https ://doi.org/10.1002/ejoc.201601226

Sangawa H, Komeno T, Nishikawa H, Yoshida A, Takahashi K, Nomura N, Furuta Y (2013) Mechanism of action of T-705 ribosyl triphosphate against influenza virus RNA polymerase. Antimicrob Agents Chemother 5711:5202-5208. https://doi.org/10.1128/aac.00649-13

Sato N, Takeuchi R (1990a) Studies on pyrazines; part 20.1 a simple synthesis of 5-substituted 2-amino-3-cyanopyrazines: useful intermediates for pteridine synthesis. Synthesis 199008:659-660

Sato N, Takeuchi R (1990b) Studies on pyrazines; Part 20.1 a simple synthesis of 5-substituted 2-amino-3-cyanopyrazines: useful intermediates for pteridine synthesis. Synthesis 199008:659-660

Smither SJ, Eastaugh LS, Steward JA, Nelson M, Lenk RP, Lever MS (2014) Post-exposure efficacy of oral T-705 (Favipiravir) against inhalational Ebola virus infection in a mouse model. Antivir Res 104:153-155. https://doi.org/10.1016/j.antiviral.2014.01.012

Wang G, Wan J, Hu Y, Wu X, Prhave M, Dyatkina N, Rajwanshi VK, Smith DB, Jekle A, Kinkade A, Symons JA, Jin Z, Deval J, Zhang Q, Tam Y, Chanda S, Blatt L, Beigelman L (2016) Synthesis and anti-influenza activity of pyridine, pyridazine, and pyrimidine C-nucleosides as favipiravir (T-705) analogues. J Med Chem 5910:4611-4624. https://doi.org/10.1021/acs.jmedchem.5b01933 